\title{
Characterization of Biochar and Humic Acid and their Effect on Soil Properties in Maize
}

\author{
P. Madhavi*, V. Sailaja, T. Ram Prakash and S.A. Hussain
}

Department of Soil Science and Agricultural Chemistry, College of Agriculture, Professor Jayashankar Telangana State Agricultural University, Hyderabad -500030, Telangana, India *Corresponding author

\section{A B S T R A C T}

\begin{tabular}{|l|}
\hline Ke y w o r d s \\
Biochar and humic \\
acid, Soil \\
properties, Maize.
\end{tabular}

A field study was carried out at college farm, College of Agriculture, Rajendranagar, Hyderabad, Andhra Pradesh during Kharif 2013 on an Alfisol to characterize the biochar and humic acid and their effect on soil properties along with application of fertilizers in maize crop. Soil had a $\mathrm{pH}$ of 7.72, EC of $0.217 \mathrm{dS} \mathrm{m}^{-1}$ and low organic carbon $(0.49 \%)$. The experiment was laid out in a Randomized Block Design and replicated thrice with three factors comprised of factor-I (fertilizers- $100 \%$ RDF and $75 \%$ RDF), Factor-II (biochar levels- 0, 5 and $7.5 \mathrm{t} \mathrm{ha}^{-1}$ ) and Factor-III (humic acid levels of 0 and $30 \mathrm{~kg} \mathrm{ha}^{-1}$ ). Biochar application showed a non-significant increase in soil $\mathrm{pH}$. Significantly higher EC was shown when $100 \%$ NPK was integrated with biochar @ $7.5 \mathrm{t} \mathrm{ha}^{-1}$. Soil CEC was significantly higher when $100 \%$ NPK was applied in combination with biochar @ $7.5 \mathrm{t} \mathrm{ha}^{-1}$ and humic acid. Such increase at later stages was not significant. Biochar elucidated a significant increase in organic carbon from 0.51 to 0.62 per cent at 30 DAS when applied at $7.5 \mathrm{t} \mathrm{ha}^{-1}$.

\section{Introduction}

Maize (Zea mays L.) is an important food and feed crop which ranks third among cereals after wheat and rice in the world. It is a multipurpose crop that provides food for humans, feed for animals (especially poultry and livestock) and raw material for the industries.

This crop has much higher grain protein content than our staple food rice. Maize is a heavy feeder of nutrients hence it is a very efficient converter of solar energy into dry matter. India is the fifth largest producer of maize in the world contributing 3 per cent of the global production (Arif et al., 2012).
Climate change and global warming have worldwide adverse consequences. Biochar production and its use in agriculture can play a key role in climate change mitigation and help improve the quality and management of waste materials coming from agriculture and forestry. Biochar is a carbonaceous material obtained from thermal decomposition of residual biomass at relatively varying temperatures and under oxygen limited conditions (pyrolysis). Biochar is currently a subject of active research worldwide because it can constitute a viable option for sustainable agriculture due to its potential as a long term sink for carbon in soil and benefits 
for crops (Alburquerque et al., 2013). The application of biochar to agricultural land is receiving increasing attention as an intervention strategy for the sequestration of carbon and as a means of improving soil quality and nutrient cycling thereby aiming at reduced fertilizer use (Richard et al., 2012). Studies suggest that biochar sequesters approximately $50 \%$ of the carbon available within the biomass feedstock being pyrolyzed (Kelsi Bracmort, 2010).

Humic acid are mainly produced from nitrogenous compounds containing decomposed amino acids and aromatic complexes (Andriesse, 1988). Those organic complexes can improve physical properties of soils due to carboxyl (-COOH) and phenolic $(-\mathrm{OH})$ groups (Lee and Bartlette, 1976).

This present investigation is planned to characterize the biochar and humic acid and their effect on soil properties along with application of fertilizers in maize crop.

\section{Material and Methods}

This experiment was conducted during kharif, 2013 at the College Farm, Acharya N.G Ranga Agricultural University, Rajendranagar, Hyderabad comes under the Southern Telangana agro-climatic zone of Andhra Pradesh. The details of the material used and the methods adopted during the course of the present investigation are given below.

The $\mathrm{pH}$ and Electrical conductivity of the soil samples were determined in soil: water (1:2) suspension using a glass electrode $\mathrm{pH}$ meter and conductivity meter respectively (Jackson, 1973). Organic carbon percentage in soil sample was determined by wet digestion method (Walkley and Black, 1934). Cation exchange capacity of soil, biochar and humic acid was determined by neutral normal ammonium acetate method.
The total acidity of biochar and humic acid was determined by Baryta absorption method Calcium acetate method was used for determining - $\mathrm{COOH}$ groups in both biochar and humic acid by calcium acetate method (Schnitzer, 1972). Biochar suspensions containing a normalized quantity of $\mathrm{C}(0.25$ g) were placed in $25 \mathrm{~mL}$ of sodium hydroxide $(0.1 \mathrm{M})$ and were stirred in a closed vessel for 20 to $24 \mathrm{hr}$. A $10 \mathrm{~mL}$ filtered aliquot was added to $15 \mathrm{~mL}$ of standard $\mathrm{HCl}(0.1 \mathrm{M})$ solution. The $\mathrm{HCl}$ neutralized the unreacted base and prevented further reaction between atmospheric $\mathrm{CO}_{2}$ and the base. The solution was then back titrated with standard $\mathrm{NaOH}$ $(0.1 \mathrm{M})$ solution. The volume of $\mathrm{NaOH}$ required to neutralize the sample was converted to titratable negative surface charge. The total negative surface charge was expressed as mmol $\mathrm{H}+\mathrm{eq} / \mathrm{g} \mathrm{C}$ (Boehm, 1994).

\section{Results and Discussion}

\section{Characterization of biochar and humic acid}

Biochar and humic acid $\mathrm{pH}$ (8.20 and 4.87), electrical conductivity (1.28 and $0.034 \mathrm{dS} \mathrm{m}^{-1}$ ), cation exchange capacity (49 and $86 \mathrm{cmol}$ $\left(\mathrm{p}^{+}\right) \mathrm{kg}^{-1}$ ), total negative charges (2.60 and $2.98 \mathrm{~m} \mathrm{~mol} \mathrm{H}^{+}$eq $\mathrm{g}^{-1}$ ), total acidity (2.5 and $3.25 \mathrm{me} \mathrm{g}^{-1}$ ) and carboxyl groups (0.2 and $3.11 \mathrm{me} \mathrm{g}^{-1}$ ) were given in table 1 .

\section{Effect of combined application of biochars, humic acid and fertilizers on some soil properties}

\section{Soil pH}

Soil $\mathrm{pH}$ was not influenced significantly by either individual or integrated application of fertilisers, biochar and humic acid at any stage of crop growth. However, a nonsignificant increase was observed with the application of biochar. The alkaline nature of the biochar exchanges $\mathrm{H}^{+}$with the 
surrounding soil, causing a rise in soil $\mathrm{pH}$. Streubel et al., (2011) elucidated a significant increase in soil $\mathrm{pH}$ by biochar application (Tables 2, 3, 4).

\section{Electrical conductivity}

Electrical conductivity of the soil shown differently at different stages of the crop. Electrical conductivity of the soil was not influenced by either individual and integrated application of fertilisers, biochar or humic acid at 30 DAS (Tables 5, 6, 7).

Whereas at 60 DAS a significant influence was exerted by individual application of fertilisers and humic acid and interaction between biochar and humic acid. Mean electrical conductivity of the soil was 0.140 $\mathrm{dS} \mathrm{m}^{-1}$ with recommended NPK as against 0.123 with reduced NPK. However, both are having normal conductivity values only. Application humic acid @ 30 kg ha ${ }^{-1}$ decreased the mean electrical conductivity of the soil significantly from 0.137 to $0.125 \mathrm{dS}$ $\mathrm{m}^{-1}$. Interaction between biochar and humic acid resulted in a significant increase in electrical conductivity of the soil to 0.152 without biochar and humic acid as against $0.116 \mathrm{dS} \mathrm{m}^{-1}$ with humic acid. Fertilisers and biochar leave salts in the soil thus increasing EC. Similar increase in EC with biochar application was elucidated by Alburquerque et al., (2013).

The electrical conductivity of the soil at harvest was influenced only by the interaction between biochar and humic acid, fertilisers with biochar and humic acid. These results are in line with the findings of Kareer et al., (2013) and Alburquerque (2013) and Nigussie et al., (2012). Interaction between biochar and humic acid resulted in a significant increase in EC from $0.239 \mathrm{dS} \mathrm{m}^{-1}$ without biochar to $0.292 \mathrm{dS} \mathrm{m}^{-1}$ with humic acid (Table 7). The interaction of fertilisers with biochar and humic acid resulted in significant increase in
EC. Reduced NPK alone resulted in significantly lower EC of $0.227 \mathrm{dS} \mathrm{m}^{-1}$ as against recommended NPK alone was significantly gave higher EC of $0.358 \mathrm{dS} \mathrm{m}^{-1}$. Between 300 to $600^{\circ} \mathrm{C}$ organic acids and phenolic substances are created and alkali salts are formed that raise the $\mathrm{pH}$ of the biochar (Shinogi and Kanri, 2003).

\section{Cation exchange capacity (CEC)}

Significantly higher mean CEC of $17.11 \mathrm{c}$ mol $\left(\mathrm{p}^{+}\right) \mathrm{kg}^{-1}$ was obtained with the application of recommended NPK as against $15.56 \mathrm{c}$ mol $\left(\mathrm{p}^{+}\right) \mathrm{kg}^{-1}$ with $75 \%$ NPK. Application of biochar brought about significant increase in the mean CEC from $15.61 \mathrm{c} \mathrm{mol}\left(\mathrm{p}^{+}\right) \mathrm{kg}^{-1}$ to $17.40 \mathrm{c} \mathrm{mol}\left(\mathrm{p}^{+}\right) \mathrm{kg}^{-}$ ${ }^{1}$ with biochar@ $0.5 \mathrm{t} \mathrm{ha}^{-1}$. However, the CEC registered in the treatments receiving 5 and $7.5 \mathrm{t} \mathrm{ha}^{-1}$ of biochar were at a par. The results corroborate the findings of Cornelissen et al., (2013) and Major et al., (2010) and Brodowski et al., (2005). Application of humic acid @ $30 \mathrm{~kg} \mathrm{ha}^{-1}$ increased the mean CEC significantly from 15.91 to $16.74 \mathrm{c} \mathrm{mol}$ $\left(\mathrm{p}^{+}\right) \mathrm{kg}^{-1}$. Similar results were obtained by Khattak et al., (2013) and Ebtisam et al., (2012).

The interaction between fertilisers and biochar was found significant. CEC significantly increased from $16.84 \mathrm{c} \mathrm{mol}\left(\mathrm{p}^{+}\right)$ $\mathrm{kg}^{-1}$ obtained with recommended NPK along with biochar@ $5 \mathrm{t} \mathrm{ha}^{-1}$ to $18.67 \mathrm{cmol}\left(\mathrm{p}^{+}\right) \mathrm{kg}^{-1}$ was obtained with recommended NPK with biochar@7.5 t ha ${ }^{-1}$ as against 15.84 with recommended NPK alone. While when there was no integration, both the fertiliser levels were at a par with regard to CEC with the corresponding values of 15.84 and $15.39 \mathrm{c}$ $\mathrm{mol}\left(\mathrm{p}^{+}\right) \mathrm{kg}^{-1}$ and at $7.5 \mathrm{t} \mathrm{ha}^{-1}$ level there was significant difference among the two levels. The interaction between fertilisers and humic acid, biochar and humic acid and fertilisers with biochar and humic acid did not influence the CEC of the soil at 30 DAS. 
Table.1 Characterization of biochar and humic acid

\begin{tabular}{|c|c|c|}
\hline Particulars & Value & Method of analysis \\
\hline \multicolumn{3}{|l|}{ A. Biochar } \\
\hline $\mathrm{pH}$ & 8.20 & $\begin{array}{l}\text { Glass Electrode pH meter, model: Elico LI } 120 \\
\text { (Jackson, 1973) }\end{array}$ \\
\hline 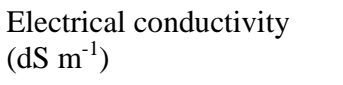 & 1.28 & $\begin{array}{l}\text { Conductivity bridge method, model: Elico CM } 180 \\
\text { (Jackson, 1973) }\end{array}$ \\
\hline 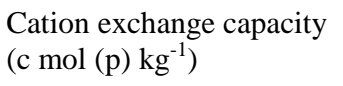 & 49 & $\begin{array}{l}\text { Neutral normal ammonium acetate method (Jackson, } \\
\text { 1973) }\end{array}$ \\
\hline $\begin{array}{l}\text { Total negative charges } \\
\left(\mathrm{m} \mathrm{mol} \mathrm{H} \mathrm{H}^{+} \text {eq } \mathrm{g}^{-1}\right)\end{array}$ & 2.60 & Boehm method (Boehm, 1994) \\
\hline $\begin{array}{l}\text { Total acidity } \\
\left(\mathrm{me}^{-1}\right)\end{array}$ & 2.5 & $\begin{array}{l}\text { Baryta absorption method } \\
\text { (Schnitzer, 1972) }\end{array}$ \\
\hline $\begin{array}{l}\text { Carboxyl groups } \\
\left(\mathrm{me}^{-1}\right)\end{array}$ & 0.2 & $\begin{array}{l}\text { Calcium acetate method } \\
\text { (Schnitzer, 1972) }\end{array}$ \\
\hline \multicolumn{3}{|l|}{ B. Humic acid } \\
\hline $\mathrm{pH}$ & 4.87 & $\begin{array}{l}\text { Glass Electrode pH meter, model: Elico LI } 120 \\
\text { (Jackson, 1973) }\end{array}$ \\
\hline 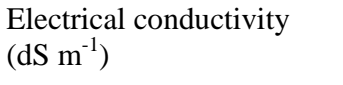 & 0.034 & $\begin{array}{l}\text { Conductivity bridge method, model: Elico CM } 180 \\
\text { (Jackson, 1973) }\end{array}$ \\
\hline $\begin{array}{l}\text { Cation exchange capacity } \\
\left(\mathrm{c} \mathrm{mol}\left(\mathrm{p}^{+}\right) \mathrm{kg}^{-1}\right)\end{array}$ & 86 & $\begin{array}{l}\text { Neutral normal ammonium acetate method (Jackson, } \\
\text { 1973) }\end{array}$ \\
\hline $\begin{array}{l}\text { Total negative charges } \\
\left(\mathrm{m} \mathrm{mol} \mathrm{H}{ }^{+} \text {eq } \mathrm{g}^{-1}\right)\end{array}$ & 2.98 & Boehm method (Boehm, 1994) \\
\hline $\begin{array}{l}\text { Total acidity } \\
\left(\mathrm{me}^{-1}\right)\end{array}$ & 3.25 & $\begin{array}{l}\text { Baryta absorption method } \\
\text { (Schnitzer, 1972) }\end{array}$ \\
\hline $\begin{array}{l}\text { Carboxyl groups } \\
\left(\mathrm{me} \mathrm{g}^{-1}\right)\end{array}$ & 3.11 & $\begin{array}{l}\text { Calcium acetate method } \\
\text { (Schnitzer, 1972) }\end{array}$ \\
\hline $\begin{array}{l}\text { Phenolic group } \\
\left(\mathrm{me}^{-1}\right)\end{array}$ & 2.02 & $\begin{array}{l}\text { Acetylation with acetic anhydride method (Stevenson, } \\
\text { 1982) }\end{array}$ \\
\hline E4/E6 & 4.86 & $\begin{array}{l}\text { Absorption method } \\
\text { (Kononova, 1966) }\end{array}$ \\
\hline
\end{tabular}

Table.2 Soil pH at 30 DAS of maize as influenced by fertiliser, biochar and humic acid levels and their interaction

\begin{tabular}{|c|c|c|c|c|c|c|c|c|c|c|}
\hline Treatments & \multicolumn{3}{|c|}{ BC@0 0 ha $^{-1}$} & \multicolumn{3}{|c|}{ BC@5.0 t ha ${ }^{-1}$} & \multicolumn{3}{|c|}{ BC@ @ $7.5 \mathrm{tha}^{-1}$} & \multirow{2}{*}{$\begin{array}{l}\text { Fertiliser } \\
\text { Mean }\end{array}$} \\
\hline Fertiliser levels & $\mathbf{H A}_{1}$ & $\mathbf{H A}_{2}$ & Mean & $\mathbf{H A}^{1}$ & $\mathbf{H A}_{2}$ & Mean & $\mathbf{H A}_{1}$ & $\mathbf{H A}_{2}$ & Mean & \\
\hline $100 \%$ NPK & 7.20 & 7.26 & 7.23 & 7.14 & 7.30 & 7.22 & 7.26 & 7.52 & 7.39 & 7.28 \\
\hline $75 \%$ NPK & 7.15 & 7.28 & 7.22 & 7.13 & 7.02 & 7.08 & 7.02 & 7.18 & 7.10 & 7.13 \\
\hline Mean & 7.18 & 7.27 & 7.22 & 7.14 & 7.16 & 7.15 & 7.14 & 7.35 & 7.25 & 7.20 \\
\hline $\mathrm{CV}(\%)$ & 3.15 & & & & & & & & & \\
\hline CD at $5 \%$ level & \multicolumn{4}{|c|}{$\begin{array}{l}\text { Fert. = N.S } \\
\text { Biochar = N.S } \\
\text { Humic acid = N.S }\end{array}$} & \multicolumn{6}{|c|}{$\begin{array}{l}\text { Fert. } \mathrm{x} \text { biochar }=\mathrm{N} . \mathrm{S} \\
\text { Fert. } \mathrm{x} \text { humic acid }=\mathrm{N} . \mathrm{S} \\
\text { Biochar } \mathrm{x} \text { humic acid }=\mathrm{N} . \mathrm{S} \\
\text { Fert. } \mathrm{x} \text { biochar } \mathrm{x} \text { humic } \mathrm{x} \text { acid }=\text { N.S }\end{array}$} \\
\hline
\end{tabular}


Table.3 Soil pH at 60 DAS of maize as influenced by fertiliser, biochar and humic acid levels and their interaction

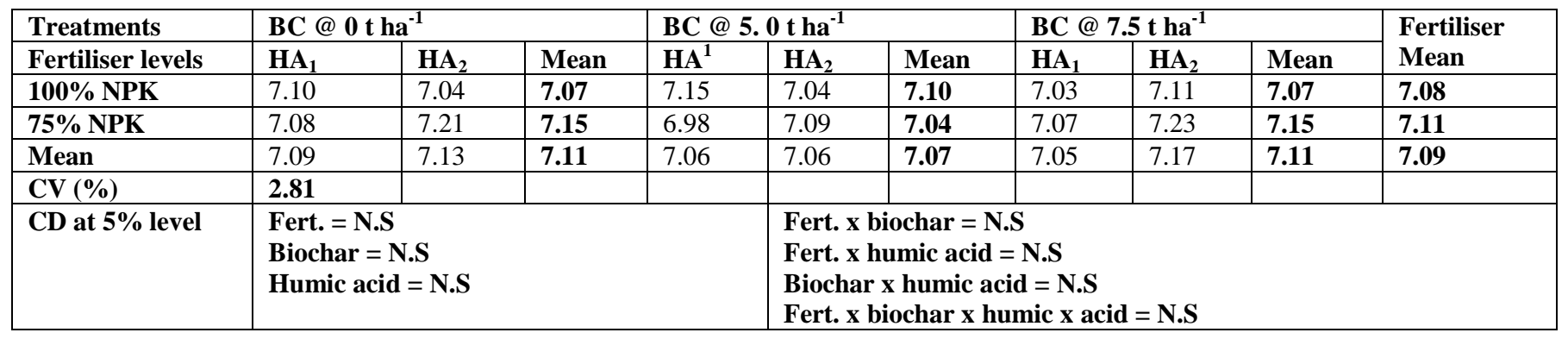

Table.4 Soil $\mathrm{pH}$ at harvest of maize as influenced by fertiliser, biochar and humic acid levels and their interaction

\begin{tabular}{|c|c|c|c|c|c|c|c|c|c|c|}
\hline Treatments & \multicolumn{3}{|c|}{ BC@0 t ha ${ }^{-1}$} & \multicolumn{3}{|c|}{ BC@ @.0 tha ${ }^{-1}$} & \multicolumn{3}{|c|}{ BC @ $7.5 \mathrm{t} \mathrm{ha}^{-1}$} & \multirow{2}{*}{$\begin{array}{l}\text { Fertiliser } \\
\text { Mean }\end{array}$} \\
\hline Fertiliser levels & $\mathbf{H A}_{1}$ & $\mathbf{H A}_{2}$ & Mean & $\mathbf{H A}^{\mathbf{1}}$ & $\mathbf{H A}_{2}$ & Mean & $\mathbf{H A}_{1}$ & $\mathbf{H A}_{2}$ & Mean & \\
\hline $100 \%$ NPK & 7.04 & 7 & 7.02 & 7.21 & 6.69 & 6.95 & 7.17 & 6.85 & 7.01 & 6.99 \\
\hline $75 \%$ NPK & 7.03 & 7.02 & 7.03 & 6.96 & 6.85 & 6.91 & 7.21 & 7.08 & 7.15 & 7.03 \\
\hline Mean & 7.04 & 7.01 & 7.02 & 7.09 & 6.77 & 6.93 & 7.19 & 6.96 & 7.08 & 7.01 \\
\hline $\mathrm{CV}(\%)$ & 4.37 & & & & & & & & & \\
\hline CD at $5 \%$ level & \multicolumn{4}{|c|}{$\begin{array}{l}\text { Fert. = N.S } \\
\text { Biochar = N.S } \\
\text { Humic acid = N.S }\end{array}$} & \multicolumn{6}{|c|}{$\begin{array}{l}\text { Fert. } \mathrm{x} \text { biochar }=\text { N.S } \\
\text { Fert. } \mathrm{x} \text { humic acid }=\text { N.S } \\
\text { Biochar } \mathrm{x} \text { humic acid }=\text { N.S } \\
\text { Fert. } \mathrm{x} \text { biochar } \mathrm{x} \text { humic } \mathrm{x} \text { acid }=\text { N.S }\end{array}$} \\
\hline
\end{tabular}

Table.5 Soil EC ( $\left.\mathrm{dS} \mathrm{m}^{-1}\right)$ at $30 \mathrm{DAS}$ of maize as influenced by fertiliser, biochar and humic acid levels and their interaction

\begin{tabular}{|c|c|c|c|c|c|c|c|c|c|c|}
\hline Treatments & \multicolumn{3}{|c|}{ BC@ @ t ha ${ }^{-1}$} & \multicolumn{3}{|c|}{ BC @ 5.0 t ha ${ }^{-1}$} & \multicolumn{3}{|c|}{ BC @ $7.5 \mathrm{t} \mathrm{ha}^{-1}$} & $\begin{array}{l}\text { Fertiliser } \\
\text { Mean }\end{array}$ \\
\hline $75 \%$ NPK & 0.192 & 0.183 & 0.187 & 0.173 & 0.184 & 0.178 & 0.162 & 0.189 & 0.175 & 0.180 \\
\hline CV (\%) & 14.03 & & & & & & & & & \\
\hline CD at $5 \%$ level & \multicolumn{4}{|c|}{$\begin{array}{l}\text { Fert. = N.S } \\
\text { Biochar = N.S } \\
\text { Humic acid = N.S }\end{array}$} & \multicolumn{6}{|c|}{$\begin{array}{l}\text { Fert. } x \text { biochar }=\text { N.S } \\
\text { Fert. } x \text { humic acid }=\text { N.S } \\
\text { Biochar } x \text { humic acid = N.S } \\
\text { Fert. } x \text { biochar } x \text { humic } x \text { acid }=\text { N.S }\end{array}$} \\
\hline
\end{tabular}

Table.6 Soil EC (dS m $\left.{ }^{-1}\right)$ at 60 DAS of maize as influenced by fertiliser, biochar and humic acid levels and their interaction

\begin{tabular}{|c|c|c|c|c|c|c|c|c|c|c|}
\hline Treatments & \multicolumn{3}{|c|}{ BC@ @ 0 t ha ${ }^{-1}$} & \multicolumn{3}{|c|}{ BC @ 9.0 t ha $^{-1}$} & \multicolumn{3}{|c|}{ BC @ 7.5 t ha $^{-1}$} & \multirow{2}{*}{$\begin{array}{l}\text { Fertiliser } \\
\text { Mean }\end{array}$} \\
\hline Fertiliser levels & $\mathbf{H A}_{1}$ & $\mathbf{H A}_{2}$ & Mean & $\mathbf{H A}^{1}$ & $\mathbf{H A}_{2}$ & Mean & $\mathbf{H A}_{1}$ & $\mathbf{H A}_{2}$ & Mean & \\
\hline $100 \%$ NPK & 0.150 & 0.122 & 0.136 & 0.120 & 0.126 & 0.123 & 0.151 & 0.170 & 0.161 & 0.140 \\
\hline $75 \%$ NPK & 0.154 & 0.119 & 0.137 & 0.155 & 0.107 & 0.131 & 0.091 & 0.110 & 0.101 & 0.123 \\
\hline Mean & 0.152 & 0.120 & 0.136 & 0.137 & 0.116 & 0.127 & 0.121 & 0.140 & 0.131 & 0.131 \\
\hline CV (\%) & 13.73 & & & & & & & & & \\
\hline CD at 5\% level & \multicolumn{4}{|c|}{$\begin{array}{l}\text { Fert. }=0.012 \\
\text { Biochar }=\text { N.S } \\
\text { Humic acid }=0.012\end{array}$} & \multicolumn{6}{|c|}{$\begin{array}{l}\text { Fert. } \mathrm{x} \text { biochar }=\text { N.S } \\
\text { Fert. } \mathrm{x} \text { humic acid }=\text { N.S } \\
\text { Biochar } \mathrm{x} \text { humic acid }=0.022 \\
\text { Fert. } \mathrm{x} \text { biochar } \mathrm{x} \text { humic } \mathrm{x} \text { acid }=\text { N.S }\end{array}$} \\
\hline
\end{tabular}


Table.7 Soil EC $\left(\mathrm{dS} \mathrm{m}{ }^{-1}\right)$ at harvest of maize as influenced by fertiliser, biochar and humic acid levels and their interaction

\begin{tabular}{|c|c|c|c|c|c|c|c|c|c|c|}
\hline Treatments & \multicolumn{3}{|c|}{ BC @ 0 t ha $^{-1}$} & \multicolumn{3}{|c|}{ BC @ $9.0 \mathrm{tha}^{-1}$} & \multicolumn{3}{|c|}{ BC @ $7.5 \mathrm{t} \mathrm{ha}^{-1}$} & $\begin{array}{l}\text { Fertiliser } \\
\text { Mean }\end{array}$ \\
\hline $75 \%$ NPK & 0.227 & 0.275 & 0.251 & 0.288 & 0.281 & 0.284 & 0.290 & 0.257 & 0.274 & 0.270 \\
\hline CV (\%) & 11.283 & & & & & & & & & \\
\hline CD at $5 \%$ level & \multicolumn{4}{|c|}{$\begin{array}{l}\text { Fert. }=\text { N.S } \\
\text { Biochar = N.S } \\
\text { Humic acid = N.S }\end{array}$} & \multicolumn{6}{|c|}{$\begin{array}{l}\text { Fert. } \mathrm{x} \text { biochar }=\text { N.S } \\
\text { Fert. } \mathrm{x} \text { humic acid }=\text { N.S } \\
\text { Biochar } \mathrm{x} \text { humic acid }=0.037 \\
\text { Fert. } \mathrm{x} \text { biochar } \mathrm{x} \text { humic } \mathrm{x} \text { acid }=0.052\end{array}$} \\
\hline
\end{tabular}

Table.8 Cation exchange capacity of soil (c mol (p) $\mathrm{kg}^{-1}$ ) at 30 DAS of maize as influenced by fertiliser, biochar and humic acid levels and their interaction

\begin{tabular}{|c|c|c|c|c|c|c|c|c|c|c|}
\hline Treatments & \multicolumn{3}{|c|}{ BC@0t ha ${ }^{-1}$} & \multicolumn{3}{|c|}{ BC@5.0 t ha ${ }^{-1}$} & \multicolumn{3}{|c|}{ BC @ $7.5 \mathrm{t} \mathrm{ha}^{-1}$} & \multirow{2}{*}{$\begin{array}{l}\text { Fertiliser } \\
\text { Mean }\end{array}$} \\
\hline Fertiliser levels & $\mathbf{H A}_{1}$ & $\mathbf{H A}_{2}$ & Mean & $\mathbf{H A}^{1}$ & $\mathbf{H A}_{2}$ & Mean & $\mathbf{H A}_{1}$ & $\mathbf{H A}_{2}$ & Mean & \\
\hline $75 \%$ NPK & 15.20 & 15.58 & 15.39 & 14.23 & 16.09 & 15.16 & 15.95 & 16.33 & 16.10 & 15.56 \\
\hline CV $(\%)$ & 4.62 & & & & & & & & & \\
\hline CD at $5 \%$ level & \multicolumn{4}{|c|}{$\begin{array}{l}\text { Fert. }=0.52 \\
\text { Biochar }=0.64 \\
\text { Humic acid }=0.52\end{array}$} & \multicolumn{6}{|c|}{$\begin{array}{l}\text { Fert. } \mathrm{x} \text { biochar }=0.90 \\
\text { Fert. } \mathrm{x} \text { humic acid }=\mathrm{N} . \mathrm{S} \\
\text { Biochar } \mathrm{x} \text { humic acid }=\mathrm{N} . \mathrm{S} \\
\text { Fert. } \mathrm{x} \text { biochar } \mathrm{x} \text { humic } \mathrm{x} \text { acid }=\text { N.S }\end{array}$} \\
\hline
\end{tabular}

Table.9 Cation exchange capacity of soil (c mol (p) $\mathrm{kg}^{-1}$ ) at 60 DAS of maize as influenced by fertiliser, biochar and humic acid levels and their interaction

\begin{tabular}{|c|c|c|c|c|c|c|c|c|c|c|}
\hline Treatments & \multicolumn{3}{|c|}{ BC @ 0 t ha $^{-1}$} & \multicolumn{3}{|c|}{ BC@5.0 $\mathrm{tha}^{-1}$} & \multicolumn{3}{|c|}{ BC@ $9.5 \mathrm{t} \mathrm{ha}^{-1}$} & \multirow{2}{*}{$\begin{array}{l}\text { Fertiliser } \\
\text { Mean }\end{array}$} \\
\hline Fertiliser levels & $\mathbf{H A}_{1}$ & $\mathbf{H A}_{2}$ & Mean & $\mathbf{H A}^{1}$ & $\mathbf{H A}_{2}$ & Mean & $\mathbf{H A}_{1}$ & $\mathbf{H A}_{2}$ & Mean & \\
\hline $75 \%$ NPK & 15.09 & 15.36 & 15.23 & 15.01 & 14.91 & 14.96 & 15.1 & 15.64 & 15.37 & 15.18 \\
\hline $\mathrm{CV}(\%)$ & 5.28 & & & & & & & & & \\
\hline CD at $5 \%$ level & \multicolumn{4}{|c|}{$\begin{array}{l}\text { Fert. = N.S } \\
\text { Biochar = N.S } \\
\text { Humic acid = N.S }\end{array}$} & \multicolumn{6}{|c|}{$\begin{array}{l}\text { Fert. } \mathrm{x} \text { biochar }=\mathrm{N} . \mathrm{S} \\
\text { Fert. } \mathrm{x} \text { humic acid }=\mathrm{N} . \mathrm{S} \\
\text { Biochar } \mathrm{x} \text { humic acid }=\text { N.S } \\
\text { Fert. } \mathrm{x} \text { biochar } \mathrm{x} \text { humic } \mathrm{x} \text { acid }=\text { N.S }\end{array}$} \\
\hline
\end{tabular}

Table.10 Cation exchange capacity ( $\mathrm{c} \mathrm{mol}(\mathrm{p}) \mathrm{kg}^{-1}$ ) of soil at harvest of maize as influenced by fertiliser, biochar and humic acid levels and their interaction

\begin{tabular}{|c|c|c|c|c|c|c|c|c|c|c|}
\hline Treatments & \multicolumn{3}{|c|}{ BC @ 0 t ha $^{-1}$} & \multicolumn{3}{|c|}{ BC@ $9.0 \mathrm{tha}^{-1}$} & \multicolumn{3}{|c|}{ BC @ $7.5 \mathrm{t} \mathrm{ha}^{-1}$} & \multirow{2}{*}{$\begin{array}{l}\text { Fertiliser } \\
\text { Mean }\end{array}$} \\
\hline & $\mathbf{H A}_{\mathbf{1}}$ & $\mathbf{H A}_{2}$ & Mean & $\mathbf{H A}^{\mathbf{1}}$ & $\mathbf{H A}_{2}$ & Mean & $\mathbf{H A}_{1}$ & $\mathbf{H A}_{2}$ & Mean & \\
\hline $75 \%$ NPK & 15.23 & 15.15 & 15.19 & 15.14 & 15.04 & 15.09 & 15.22 & 15.08 & 15.15 & 15.14 \\
\hline CV (\%) & 4.36 & & & & & & & & & \\
\hline CD at $5 \%$ level & \multicolumn{4}{|c|}{$\begin{array}{l}\text { Fert. = N.S } \\
\text { Biochar = N.S } \\
\text { Humic acid = N.S }\end{array}$} & \multicolumn{6}{|c|}{$\begin{array}{l}\text { Fert. } \mathrm{x} \text { biochar }=\text { N.S } \\
\text { Fert. } \mathrm{x} \text { humic acid }=\text { N.S } \\
\text { Biochar } \mathrm{x} \text { humic acid }=\text { N.S } \\
\text { Fert. } \mathrm{x} \text { biochar } \mathrm{x} \text { humic } \mathrm{x} \text { acid }=\text { N.S }\end{array}$} \\
\hline
\end{tabular}


Table.11 Soil OC (\%) at 30 DAS of maize as influenced by fertiliser, biochar and humic acid levels and their interaction

\begin{tabular}{|c|c|c|c|c|c|c|c|c|c|}
\hline Treatments & \multicolumn{3}{|c|}{ BC@ @ t ha ${ }^{-1}$} & \multicolumn{3}{|c|}{ BC@5.0 t ha-1 } & \multicolumn{3}{|c|}{ BC@ $9.5 \mathrm{tha}^{-1}$} \\
\hline & $\mathbf{H A}_{1}$ & $\mathbf{H A}_{2}$ & Mean & $\mathbf{H A}^{1}$ & $\mathbf{H A}_{2}$ & Mean & $\mathbf{H A}_{1}$ & $\mathbf{H A}_{2}$ & Mean \\
\hline $100 \%$ NPK & 0.48 & 0.56 & 0.52 & 0.59 & 0.61 & 0.60 & 0.61 & 0.60 & 0.61 \\
\hline $75 \%$ NPK & 0.47 & 0.51 & 0.49 & 0.58 & 0.60 & 0.59 & 0.62 & 0.63 & 0.63 \\
\hline Mean & 0.48 & 0.54 & 0.51 & 0.59 & 0.61 & 0.60 & 0.62 & 0.62 & 0.62 \\
\hline CV (\%) & 16.87 & & & & & & & & \\
\hline CD at $5 \%$ level & \multicolumn{4}{|c|}{$\begin{array}{l}\text { Fert. }=\text { N.S } \\
\text { Biochar }=0.05 \\
\text { Humic acid = N.S }\end{array}$} & \multicolumn{5}{|c|}{$\begin{array}{l}\text { Fert. } \mathrm{x} \text { biochar }=\text { N.S } \\
\text { Fert. } \mathrm{x} \text { humic acid }=\text { N.S } \\
\text { Biochar } \mathrm{x} \text { humic acid }=\text { N.S } \\
\text { Fert. } \mathrm{x} \text { biochar } \mathrm{x} \text { humic } \mathrm{x} \text { acid }=\text { N.S }\end{array}$} \\
\hline
\end{tabular}

Table.12 Soil OC (\%) at 60 DAS of maize as influenced by fertiliser, biochar and humic acid levels and their interaction

\begin{tabular}{|c|c|c|c|c|c|c|c|c|c|}
\hline Treatments & \multicolumn{3}{|c|}{ BC @ o t ha ${ }^{-1}$} & \multicolumn{3}{|c|}{ BC@ @ 5.0 tha ${ }^{-1}$} & \multicolumn{3}{|c|}{ BC @ $7.5 \mathrm{tha}^{-1}$} \\
\hline & $\mathbf{H A}_{1}$ & $\mathbf{H A}_{2}$ & Mean & $\mathbf{H A}^{1}$ & $\mathbf{H A}_{2}$ & Mean & $\mathbf{H A}_{1}$ & $\mathbf{H A}_{2}$ & Mean \\
\hline $100 \%$ NPK & 0.53 & 0.57 & 0.55 & 0.6 & 0.66 & 0.63 & 0.64 & 0.65 & 0.65 \\
\hline $75 \%$ NPK & 0.59 & 0.66 & 0.63 & 0.69 & 0.63 & 0.66 & 0.69 & 0.68 & 0.69 \\
\hline Mean & 0.56 & 0.62 & 0.59 & 0.64 & 0.65 & 0.65 & 0.66 & 0.67 & 0.67 \\
\hline CV (\%) & 8.62 & & & & & & & & \\
\hline CD at $5 \%$ level & \multicolumn{4}{|c|}{$\begin{array}{l}\text { Fert. }=\text { N.S } \\
\text { Biochar }=\text { N.S } \\
\text { Humic acid = N.S }\end{array}$} & \multicolumn{5}{|c|}{$\begin{array}{l}\text { Fert. } \mathrm{x} \text { biochar }=\text { N.S } \\
\text { Fert. } \mathrm{x} \text { humic acid }=\text { N.S } \\
\text { Biochar } \mathrm{x} \text { humic acid }=\text { N.S } \\
\text { Fert. } \mathrm{x} \text { biochar } \mathrm{x} \text { humic } \mathrm{x} \text { acid }=\text { N.S }\end{array}$} \\
\hline
\end{tabular}

Table.13 Soil OC (\%) at harvest of maize as influenced by fertiliser, biochar and humic acid levels and their interaction

\begin{tabular}{|c|c|c|c|c|c|c|c|c|c|}
\hline Treatments & \multicolumn{3}{|c|}{ BC@0 t ha ${ }^{-1}$} & \multicolumn{3}{|c|}{ BC@ $9.0 \mathrm{t} \mathrm{ha}^{-1}$} & \multicolumn{3}{|c|}{ BC @ $7.5 \mathrm{t} \mathrm{ha}^{-1}$} \\
\hline & $\mathbf{H A}_{1}$ & $\mathbf{H A}_{2}$ & Mean & $\mathbf{H A}^{1}$ & $\mathbf{H A}_{2}$ & Mean & $\mathbf{H A}_{1}$ & $\mathbf{H A}_{2}$ & Mean \\
\hline $100 \%$ NPK & 0.59 & 0.59 & 0.59 & 0.6 & 0.63 & 0.62 & 0.68 & 0.67 & 0.68 \\
\hline $75 \%$ NPK & 0.54 & 0.57 & 0.56 & 0.65 & 0.63 & 0.64 & 0.66 & 0.68 & 0.67 \\
\hline Mean & 0.57 & 0.58 & 0.57 & 0.63 & 0.63 & 0.63 & 0.67 & 0.67586 & 0.67 \\
\hline $\mathrm{CV}(\%)$ & 12.58 & & & & & & & & \\
\hline CD at $5 \%$ level & \multicolumn{4}{|c|}{$\begin{array}{l}\text { Fert. }=\text { N.S } \\
\text { Biochar }=\text { N.S } \\
\text { Humic acid = N.S }\end{array}$} & \multicolumn{5}{|c|}{$\begin{array}{l}\text { Fert. } \mathrm{x} \text { biochar }=\text { N.S } \\
\text { Fert. } \mathrm{x} \text { humic acid }=\text { N.S } \\
\text { Biochar } \mathrm{x} \text { humic acid }=\text { N.S } \\
\text { Fert. } \mathrm{x} \text { biochar } \mathrm{x} \text { humic } \mathrm{x} \text { acid }=\text { N.S }\end{array}$} \\
\hline \multicolumn{5}{|c|}{$\begin{array}{l}100 \% \text { NPK: } 180-60-50 \mathrm{~N}^{-} \mathrm{P}_{2} \mathrm{O}_{5}-\mathrm{K}_{2} \mathrm{O} \mathrm{kg} \mathrm{ha}^{-1} \\
75 \% \text { NPK: } 135-45-37.5 \mathrm{~N}^{-} \mathrm{P}_{2} \mathrm{O}_{5}-\mathrm{K}_{2} \mathrm{O} \mathrm{kg} \mathrm{ha}\end{array}$} & \multicolumn{5}{|c|}{$\begin{array}{l}\mathrm{HA}_{1}: 0 \mathrm{~kg} \mathrm{ha}^{-1} \\
\mathrm{HA}_{2}: 30 \mathrm{~kg} \mathrm{ha}^{-1}\end{array}$} \\
\hline
\end{tabular}

Beneficial characteristics of biochar as a soil amendment are its high cation exchange capacity (CEC of 40 to 80 me per $100 \mathrm{~g}$ ), high surface area (51 to $900 \mathrm{~m}^{2} \mathrm{~g}^{-1}$ ), which leads to increased soil $\mathrm{pH}$ and water holding capacity, and affinity for micro and macro plant nutrients (Lehmann, 2007; Laird et al., 2009; Novak et al., 2010). When soil was amended with charcoal, annual crop yields increased by
$100 \%$ or more (Steiner et al., 2007). Cation exchange capacity was not influenced significantly by either individual or integrated application of fertilisers, biochar or humic acid at 60 DAS and at harvest (Tables 8, 9, 10). When biochar is added to the soil, due to its alkaline nature and high $\mathrm{CEC}$ it increases the CEC of the soil instantly. However, with passage of time, soil buffering capacity will 
again lower the $\mathrm{pH}$ bringing the CEC to equilibrium state (Yamato et al., 2006).

\section{Organic carbon (\%)}

Individual application of fertiliser and humic acid levels did not influence significantly the organic carbon content of the soil whereas biochar application brought about significant increase in the mean OC from 0.51 to $0.62 \%$. Biochar since is a carbonaceous material having wide $\mathrm{C}$ : $\mathrm{N}$ ratio adds organic carbon to the soil (Widowati, 2012). Interaction between fertilisers and biochar, fertilisers and humic acid, biochar and humic acid, fertilisers with biochar and humic acid were found nonsignificant. There was no significant influence of either individual and integrated application of fertilisers, biochar or humic acid on OC of the soil at 60 DAS and also at harvest (Tables $11,12,13)$.

Soil $\mathrm{pH}$ was not influenced significantly by either individual or integrated application of fertilisers, biochar and humic acid at any stage of crop growth. However, a nonsignificant increase was observed with the application of biochar. Recommended NPK and biochar at $7.5 \mathrm{t} \mathrm{ha}^{-1}$ brought about a significant increase in EC of the soil during the initial stages. Cation exchange capacity of the soil was significantly increased by humic acid@30 kg ha ${ }^{-1}$ and biochar at $7.5 \mathrm{t} \mathrm{ha}^{-1}$ at all the stages of growth, however, the effect was significant 30 DAS. Biochar application brought about significant increase in the mean OC from 0.51 to $0.62 \%$.

\section{References}

Alburquerque, J.A., Salazar, P., Barron, V., Torrent, J., Campillo, M.C., Gallardo, A and Villar, R. 2013. Enhanced wheat yield by biochar addition under different mineral fertilization levels. Agronomy for Sustainable Development. 33: 475-484.

Andriesse, J.P., 1988. Nature and management of tropical peat soils. FAO soils Bulletin No. 59, United Nations, Rome, 165.

Arif, M., Ali, A., Umair, M., Munsif, F., Ali, K., Inamullah., Saleem, M and Ayub, G. 2012. Effect of biochar, FYM and mineral nitrogen alone and in combination on yield and yield components of Maize. Sarhad Journal of Agriculture. 28(2): 191-195.

Brodowski, S., Amelung, W., Haumaier, L., Abetz, C and Zec, W. 2005. Morphological and chemical properties of black carbon in physical soil fractions as revealed by scanning electron microscopy and energy-dispersive X-ray spectroscopy. Geoderma. 128: 116-129.

Cornelissen, G., Martinsen, V., Shitumbanuma, V., Alling, V., Breedveld, G.D., Rutherford, D.W., Sparrevik, M., Hale, S.E., Obia, A and Mulder, J. 2013. Biochar effect on maize yield and soil characteristics in five conservation farming sites in Zambia. Agronomy. 3: 256-274.

Ebtisam, I.E., Sabreen, K.P., and Hady, M.A.E. 2012. Improving soil properties, maize yield components grown in sandy soil under irrigation treatments and humic acid application. Australian Journal of Basic and Applied Sciences, 6(7): 587593.

Jackson, M.L., 1973. Soil Chemical Analysis. Prentice Hall of Inco. New York, USA. 498.

Karer, J., Wimmer, B., Zehetner, F., Kloss, S and Soja, G. 2013. Biochar application to temperate soils: Effects on nutrient uptake and crop yield under field conditions. Agricutural and Food Science. 22: 390403.

Kelsi Bracmort, 2010. Biochar: Examination of an emerging concept to mitigate climate change. Congressional Research Service. 7: 5700 .

Khattak, R.A., Haroon, K and Muhammad, D. 2013. Mechanism(s) of humic acid induced beneficial effects in salt-affected soils. Scientific Research and Essays. 8(21): 932-939. 
Laird, D.A., Fleming, P., Davis, D.D. Horton, R., Wang, B.Q and Karlen, D.L. 2009. Impact of biochar amendments on the quality of a typical mid-western agricultural soil. Geoderma. 158: 443449.

Lee, Y.S., Bartlette, R. J. 1976. Stimulation of plant growth by humic substances. Soil Science of America Journal. 40: 876-879.

Lehmann, J. 2007. Bioenergy in the black. Frontiers in Ecology and the Environment. 5, 381-387.

Major, J., Rondon, M., Molina, D., Riha, S.J and Lehmann, J. 2010. Maize yield and nutrition during 4 years after biochar application to a Colombian savanna Oxisol. Plant and soil. 333: 117-128.

Nigussie, A., Kissi, E., Misganaw, $M$ and Ambaw, G. 2012. Effect of Biochar Application on Soil Properties and Nutrient Uptake of Lettuces (Lactuca sativa) Grown in Chromium Polluted Soils. American-Eurasian Journal of Agriculture \& Environmental Science. 12 (3): 369-376.

Novak, J.M., Busscher, W.J., Laird, D.L., Ahmedna, M.A., Watts, D.W and Niandou, M.A.S. 2010. Impact of biochar amendment on fertility of a southeastern coastal plain. Soil Science. 174: 105-111.

Richard, S.Q., Karina, A.M., Christoph, G., Johannes, R., Thomas, H.D and Davey, L.J. 2012. Nutrient dynamics, microbial growth and weed emergence in biochar amended soil are influenced by time since application and reapplication rate. Agriculture, Ecosystems and Environment. 158: 192-199.

Shinogi, Y., and Kanri, Y. 2003. Pyrolysis of plant, animal and human waste: physical and chemical characterization of the pyrolytic products. Bioresource Technology. 90: 241-247.

Steiner, C., Teixeira, W.G., Lehmann, J., Nehls, t., Luis, J., Macedo, L.V.D., Blum, W.E.H and Zech, W. 2007. Long term effects of manure, charcoal and mineral fertilization on crop production and fertility on a highly weathered central Amazonian upland soil. Plant and Soil. 291: 275-290.

Streubel, J.D., Collins, H.P., Garcia-Perez, M., Tarara, J., Granatstein, D, and Kruger, C.E. 2011. Influence of Biochar on Soil $\mathrm{pH}$, Water Holding Capacity, Nitrogen and Carbon Dynamics. Soil Science Society of America Journal. (In Press).

Walkley, A., and Black, C.A. 1934. Estimation of organic carbon by chromic acid titration method. Soil Science. 37: 29-38. Watanabe and Olsen, P. 1965. Methods of soil analysis-chemical and microbiological properties. Soil Science Society of America Incorporation, Medison, Wisconsin, USA.

Widowati Utomo, W.H., Guritno, B and Soehono, L.A. 2012. The effect of biochar on the growth and $\mathrm{N}$ fertilizer requirement of Maize (Zea mays L.) in green house experiment. Journal of Agricultural Science. 4(5): 255-262.

Yamato, M., Okimori, Y., Wibowo, I.F., Anshiori, S and Ogawa, M. 2006. Effects of application of charred bark of Acacia mangium on the yield of maize, cowpea and peanut and soil chemical properties in South Sumatra, Indonesia. Soil Science Plant Nutrition. 52: 489-495.

\section{How to cite this article:}

Madhavi, P., V. Sailaja, T. Ram Prakash and Hussain, S.A. 2017. Characterization of Biochar and Humic Acid and Their Effect on Soil Properties in maize. Int.J.Curr.Microbiol.App.Sci. 6(9): 449457. doi: https://doi.org/10.20546/ijcmas.2017.609.054 